\title{
AVALIAÇÃO DA EFICIÊNCIA DE REMOÇÃO DO CROMO HEXAVALENTE ATRAVÉS DA ELETROCOAGULAÇÃO
}

\author{
R. R. M. da SILVA ${ }^{1}$, I. de F. S. VIDAL ${ }^{1}$, S. C. M. SILVA ${ }^{1}$, A. C. A. M. LIMA ${ }^{1}$, \\ L.Z.MELGAR ${ }^{1}$ \\ ${ }^{1}$ Universidade Federal de São João del-Rei, Departamento de Engenharia Química \\ E-mail para contato: robertaresende38@gmail.com
}

\begin{abstract}
RESUMO - A busca por técnicas alternativas de baixo custo e eficientes na remoção de metais pesados tem se intensificado nos últimos anos. Neste trabalho investigou-se a eficiência na remoção de íons $\mathrm{Cr}(\mathrm{VI})$ por meio da aplicação da eletrocoagulação, avaliando-se os efeitos da concentração inicial de cromo hexavalente $\left(10,0 ; 25,0\right.$ e 40,0 mg. $\left.\mathrm{L}^{-1}\right)$, pH inicial $(3,0 ; 5,0$ e 9,0) e densidade de corrente $\left(0,3 ; 1,6\right.$ e $\left.2,9 \mathrm{~mA} \cdot \mathrm{cm}^{-2}\right)$ através do planejamento experimental. Os resultados estatísticos demonstraram que houve diminuição considerável na concentração inicial de cromo em todos os experimentos, nos quais a porcentagem de remoção variou entre 52,3\% - 99,77\%. Verificou-se que a densidade de corrente e a concentração inicial foram os fatores mais importantes na remoção de cromo, assim como seus efeitos de interação. Entretanto, a variação do $\mathrm{pH}$ foi insignificante. A análise estatística evidenciou uma distribuição normal dos dados.
\end{abstract}

\section{INTRODUÇÃO}

O cromo hexavalente está presente em efluentes das indústrias de galvanoplastia, curtume, fertilizantes, cimento, entre outras (Mella et al., 2013). Trata-se de uma espécie química não-biodegradável, tóxica e bioacumulativa, sendo mutagênica para os animais e potencialmente danosa para a saúde humana (Khalaf, 2016).

Os métodos existentes para o tratamento de efluentes contendo esse poluente, tornam-se inviáveis na prática por apresentarem alto custo, ou produzirem grandes volumes de lama e baixa remoção do metal, sendo, portanto, necessária uma tecnologia alternativa (Ahmaruzzaman, 2011). Dentre as disponíveis destaca-se a eletrocoagulação (EC).

A eletrocoagulação é uma técnica que utiliza a passagem de corrente elétrica para remover poluentes de uma solução. O processo envolve a geração de coagulantes pela dissolução elétrica de íons metálicos liberados no ânodo e a produção de bolhas de gás hidrogênio no cátodo, as quais auxiliam no carreamento do poluente até à superfície da solução, onde o mesmo será removido. Os eletrodos são mais comumentes constituídos por alumínio ou ferro (Al-Shannag et. al., 2013).

O método eletroquímico é simples, não dispendioso, rápido, de fácil operação e ambientalmente correto. Assim, esse método tem obtido sucesso quando aplicado para remoção de espécies iônicas solúveis e metais pesados de soluções, como o íon cromo hexavalente (Bazrafshan et al., 2015). Neste contexto, este trabalho investigou a eficiência de remoção de 
íons $\mathrm{Cr}(\mathrm{IV})$ de um efluente sintético através da técnica de eletrocoagulação utilizando-se eletrodos de alumínio. Além disso, o efeito do pH inicial da solução, da concentração inicial de cromo hexavalente e da densidade de corrente foram analisados.

\section{METODOLOGIA EXPERIMENTAL}

\subsection{Sistema de Eletrocoagulação}

Foi construído um reator de bancada de acrílico com volume de 1,3 L. Neste reator, foram inseridos 6 eletrodos de alumínio arranjados de forma monopolar em paralelo com dimensões de $8 \times 13 \times 0,1 \mathrm{~cm}$, sendo estes, conectados a uma fonte de corrente contínua (HIKARI HF3205S), como ilustra a Figura 1. A distância entre os eletrodos foi de $1 \mathrm{~cm}$. O reator foi mantido sob uma placa de agitação (THELGA) e agitadores magnéticos foram utilizados para promover a homogeneização do meio e flotação do contaminante. Durante os ensaios experimentais, a temperatura foi monitorada, a fim de assegurar-se que não haveria dissipação de energia para o meio. O tempo de EC foi de 15 minutos.

Figura 1 - a) Sistema de eletrocoagulação; b) configuração dos eletrodos.

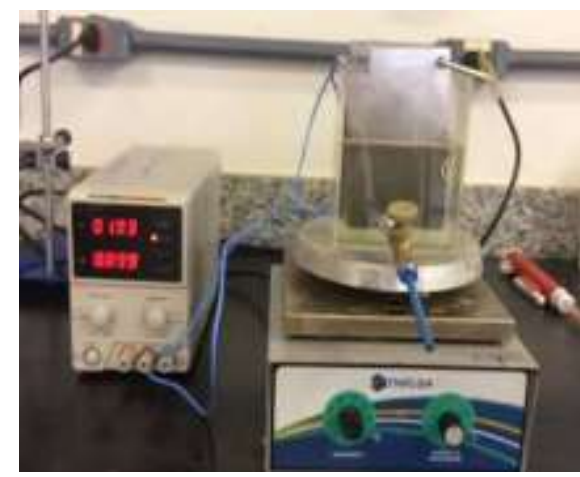

(a)

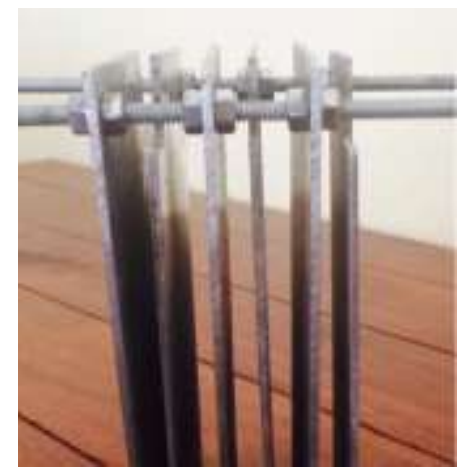

(b)

\subsection{Reagentes e Soluções}

Preparou-se uma solução $40 \mathrm{mg} . \mathrm{L}^{-1}$ de dicromato de potássio anidro (99 \% PA-ACS, Alphatec). Para a obtenção das soluções de concentrações menores, estas foram preparadas por diluição utilizando-se água deionizada. Para a realização dos experimentos adicionou-se 2,7 mmol.L $\mathrm{L}^{-1}$ do eletrólito $\mathrm{NaCl}(99,5 \%$ PA-ACS, Alphatec). O ajuste do pH foi feito utilizando-se ácido clorídrico (95-99\% PA, Vetec), 0,1 mol.L ${ }^{-1}$, e hidróxido de sódio (99 \% PA, CRQ Produtos Químicos), 0,1 mol.L ${ }^{-1}$. A quantificação de cromo hexavalente presente no efluente tratato foi feita a partir da técnica de espectrometria UV-Visível, de acordo com o Standard Methods número 3500 - Cr. A curva de calibração e as análises do efluente tratado foram feitas utilizando-se o kit de reagentes Spectro Kit Cromo (Alfakit).

\subsection{Planejamento Experimental}

A fim de determinar quais parâmetros (concentração inicial de cromo hexavalente, $\mathrm{pH}$ inicial e densidade de corrente) influenciam na remoção de $\operatorname{Cr}(\mathrm{VI})$ através da eletroflotação, 
um planejamento fatorial completo (PFC) foi utilizado. O número total de experimentos em dois níveis $(+1,-1)$ necessários para a investigação foi de 13 , sendo $2^{3}$ referentes ao fatorial completo e 5 pontos centrais, os quais foram adicionados a fim de se verificar a variância dentro dos experimentos (Montgomery, 2013). Na Tabela 1 estão apresentadas as variáveis codificadas e reais da matriz de planejamento e os níveis das variáveis.

Tabela 1 - Níveis e variáveis de estudo

\begin{tabular}{|c|c|c|c|}
\hline Níveis & $\begin{array}{c}\text { Concentração inicial de } \\
\text { Cr(VI) }\left(\mathrm{mg}^{-1}\right)\end{array}$ & $\mathrm{pH}$ & $\begin{array}{c}\text { Densidade de corrente } \\
\left(\mathrm{mA} / \mathrm{cm}^{2}\right)\end{array}$ \\
\hline-1 & 10,0 & 5,0 & 0,3 \\
\hline 0 & 25,0 & 7,0 & 1,6 \\
\hline 1 & 40,0 & 9,0 & 2,9 \\
\hline
\end{tabular}

\section{RESULTADOS E DISCUSSÃO}

\subsection{Resultados dos Ensaios de Eletrocoagulação}

$\mathrm{Na}$ Tabela 2 são apresentados os dados de eficiência de remoção (RE\%) de cromo hexavalente obtidos nos ensaios de eletrocoagulação, assim como seus respectivos valores de consumo de energia elétrica. A eficiência de remoção de íon $\mathrm{Cr}(\mathrm{VI})$ em cada experimento foi calculada a partir da Equação 1, onde C é a concentração final $\left(\mathrm{mg} \cdot \mathrm{L}^{-1}\right)$ e $\mathrm{C}_{0}$ a concentração inicial de cromo hexavalente (mg. $\left.\mathrm{L}^{-1}\right)$.

$$
R E \%=\frac{\left(C_{0}-C\right)}{C_{0}} \times 100
$$

O consumo de energia elétrica (E) foi obtido a partir da Equação 2, onde U é a tensão elétrica aplicada no sistema (V); i é a corrente elétrica (A); t é o tempo de aplicação da corrente (h); V é o volume de efluente tratado $\left(\mathrm{m}^{3}\right)$ (Mella et al., 2013).

$$
\mathrm{E}=\frac{U . i . t}{V}
$$

Tabela 2 - Matriz padrão para o planejamento fatorial completo e resultados obtidos

\begin{tabular}{|c|c|c|c|c|c|}
\hline Experimentos & $\mathrm{pH}$ & $\begin{array}{c}\text { Densidade de } \\
\text { corrente } \\
\left(\mathrm{mA} \cdot \mathrm{cm}^{-2}\right)\end{array}$ & $\begin{array}{c}\text { Concentração } \\
\text { inicial de Cr(VI) } \\
\left(\mathrm{mg}^{-1}\right)\end{array}$ & $\begin{array}{c}\text { Eficiência } \\
\text { de remoção } \\
(\%)\end{array}$ & $\begin{array}{c}\text { Energia elétrica } \\
\text { consumida } \\
\left(\mathrm{kWh} / \mathrm{m}^{3}\right)\end{array}$ \\
\hline 1 & -1 & -1 & -1 & 99,00 & 0,01 \\
\hline 2 & 1 & -1 & -1 & 99,75 & 0,01 \\
\hline 3 & -1 & 1 & -1 & 99,51 & 0,62 \\
\hline 4 & 1 & 1 & -1 & 99,51 & 0,58 \\
\hline 5 & -1 & -1 & 1 & 52,30 & 0,01 \\
\hline 6 & 1 & -1 & 1 & 52,30 & 0,01 \\
\hline 7 & -1 & 1 & 1 & 99,74 & 0,63 \\
\hline 8 & 1 & 1 & 1 & 99,49 & 0,39 \\
\hline 9 & 0 & 0 & 0 & 99,76 & 0,17 \\
\hline 10 & 0 & 0 & 0 & 99,51 & 0,17 \\
\hline 11 & 0 & 0 & 0 & 99,77 & 0,16 \\
\hline 12 & 0 & 0 & 0 & 99,69 & 0,17 \\
\hline 13 & 0 & 0 & 99,71 & 0,17 \\
\hline
\end{tabular}


A concentração máxima de descarte permitida pela resolução $n^{\circ} 430 / 2011$ do CONAMA para o cromo hexavalente é de $0,1 \mathrm{mg} . \mathrm{L}^{-1}$. Dos resultados da Tabela 2, observa-se que houve uma redução na concentração de íon $\mathrm{Cr}(\mathrm{VI})$ inicial considerável em todas as análises, sendo que os experimentos $1,2,3,4,7,9,11,12$ e 13 se adequaram à legislação. Os ensaios restantes não atingiram as exigências, apesar de apresentarem cerca de $50-99 \%$ de remoção do poluente. Com relação ao consumo de energia, os valores são aceitáveis e variam entre 0,01 $0,63 \mathrm{kWh} / \mathrm{m}^{3}$.

\subsection{Resultados da Análise Estatística dos Dados}

$\mathrm{Na}$ Tabela 3 são apresentados os efeitos estimados e os valores de p-value das variáveis do PFC $2^{3}$ sobre os resultados de remoção de cromo hexavalente, onde verifica-se que a densidade de corrente, a concentração inicial de $\mathrm{Cr}(\mathrm{VI})$ e as interações de segunda ordem foram significativas sobre o processo de eletrocoagulação $(\mathrm{p}<0,05)$.

Tabela 3- Efeitos estimados e os coeficientes de regressão das variáveis do PFC sobre os resultados de remoção de cromo hexavalente

\begin{tabular}{|l|c|c|}
\hline \multicolumn{1}{|c|}{ Fonte de variação } & $\begin{array}{c}\text { Efeitos } \\
\text { Estimados }\end{array}$ & $\begin{array}{c}\text { Nível de significânica (p- } \\
\text { value) }\end{array}$ \\
\hline $\mathrm{pH}\left(\mathrm{x}_{1}\right)$ & 0,13 & 0,167 \\
\hline Densidade de corrente $\left(\mathrm{x}_{2}\right)$ & 23,73 & 0,000 \\
\hline Concentração inicial $\left(\mathrm{x}_{3}\right)$ & $-23,49$ & 0,000 \\
\hline $\mathrm{pH}$ x densidade de corrente $\left(\mathrm{x}_{1} \mathrm{x}_{2}\right)$ & $-0,25$ & 0,027 \\
\hline $\mathrm{pH}$ x Concentração inicial $\left(\mathrm{x}_{1} \mathrm{x}_{3}\right)$ & $-0,25$ & 0,027 \\
\hline Densidade de corrente $\mathrm{x}$ concentração inicial $\left(\mathrm{x}_{2} \mathrm{x}_{3}\right)$ & 23,59 & 0,000 \\
\hline
\end{tabular}

De acordo com os resultados exibidos na Tabela 3, a densidade de corrente foi o parâmetro mais significante nesse processo, uma vez que ela apresenta maior valor de tamanho do efeito sobre a eficiência de remoção de cromo $(23,73)$. O sinal positivo do efeito indica que a remoção do íon é mais eficiente com o aumento da densidade de corrente. Esse fato se justifica, uma vez que quanto mais alta a densidade de corrente, eleva-se as taxas de geração de bolhas e de metal oxidado, resultando em uma maior geração de gases e quantidade de flocos de agente coagulante produzidos, favorecendo a flotação dos resíduos. Além disso, ao se aplicarem altos valores de densidade de corrente, há uma melhoria na eficiência do processo e uma redução no tempo operacional. O sinal negativo da concentração inicial supõe que menores valores de concentração favorecem a eficiência do processo. O que já era esperado, uma vez que quanto menor a concentração, menor a quantidade de íons do poluente dispersos em solução (El-Nass et al., 2014). O efeito de interação entre essas variáveis mostra que quando a densidade de corrente diminui e a concentração de cromo aumenta, a eficiência do processo é reduzida. Por outro lado, o aumento da densidade de corrente até $2,9 \mathrm{~mA} \cdot \mathrm{cm}^{-2}$ e a redução da concentração, favorecem no aumento da taxa de remoção do poluente. A variação do pH inicial foi insignificante. $\mathrm{O}$ mesmo resultado foi obtido em outros estudos presentes na literatura (Fornari, 2007; Casqueira et al., 2006), nos quais experimentos com diferentes valores de $\mathrm{pH}$ submetidos as mesmas condições operacionais mostraram pouca influência no aumento da eficiência de remoção de cromo. Outros metais como ferro e zinco demonstraram o mesmo comportamento, sendo este fato correlacionado a característica de alta condutividade do 
efluente que favorece o processo eletrolítico e torna o processo pouco dependente das condições de $\mathrm{pH}$ do meio.

O gráfico de Pareto, abaixo, mostra que todos os efeitos que ultrapassam a linha de referência representada pelo valor de $F_{\text {crítico }}(2,8)$ são significantes, desse modo pode-se confirmar, mais uma vez, que o efeito da densidade de corrente, da concentração inicial de cromo hexavalente (C) e das interações secundárias são significantes para a remoção.

Figura 2 - Gráfico de pareto para os efeitos principais e de interação.

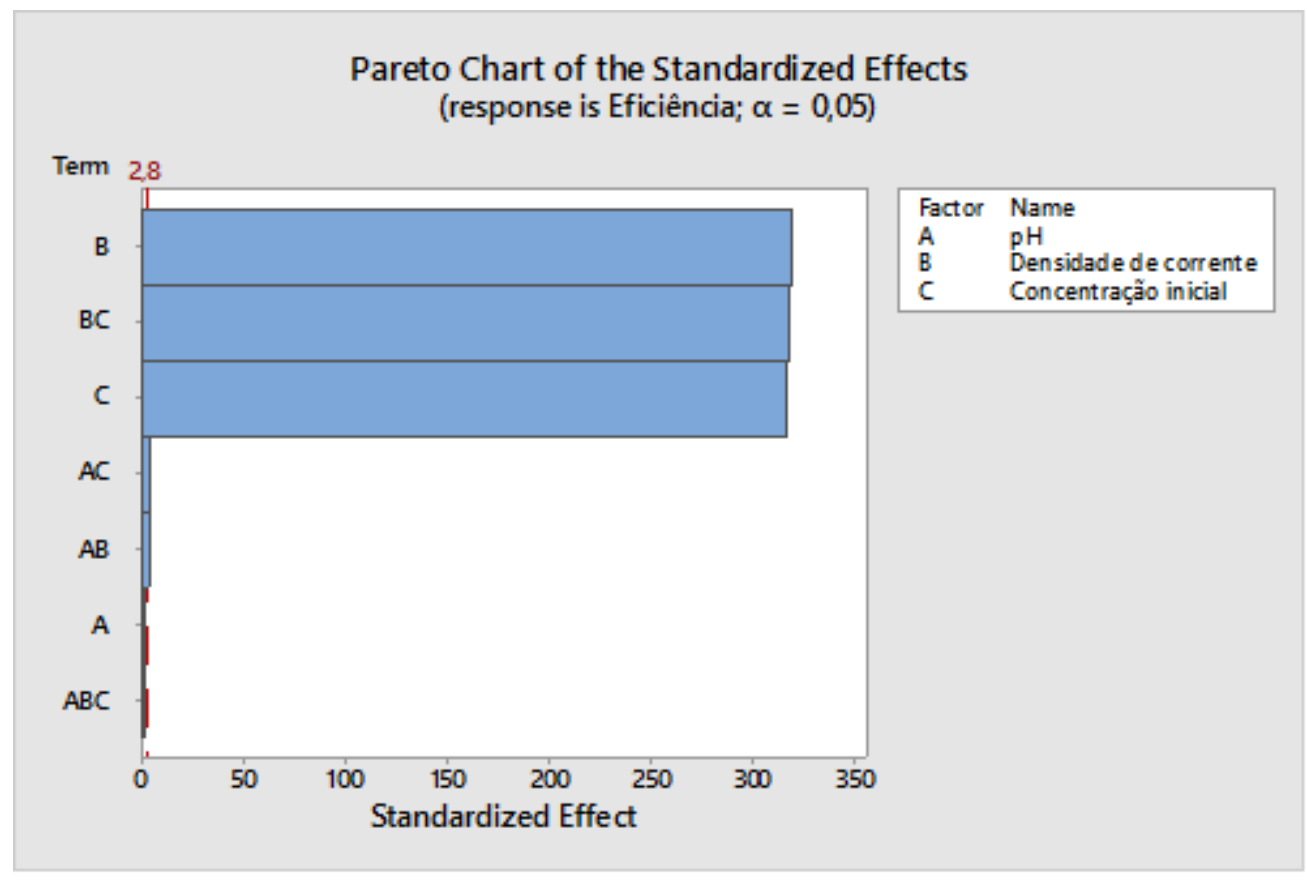

O teste de normalidade dos resíduos a partir do método de Anderson-Darling indicou que os resíduos estão distribuídos de forma normal $(p>0,05)$, contextualizando que os experimentos foram feitos de forma coerente.

\section{CONCLUSÕES}

O planejamento fatorial se mostrou um método satisfatório para determinar os principais fatores que afetam na eficiência de remoção de cromo no processo de eletrocoagulação. A densidade de corrente foi o parâmetro que mais influenciou na remoção de cromo, quanto mais alta a densidade de corrente, melhor foi a efiência de remoção. Além disso, as interações secundárias e a concentração inicial de Cr (VI) também foram fatores significantes, sendo que o aumento da concentração provocou efeito negativo na eficiência do processo. O teste de normalidade dos resíduos indicou que os resíduos estão distribuídos de forma normal, contextualizando que os experimentos foram feitos de forma coerente. A aplicação da eletrocoagulação foi satisfatória, caracterizando-se como uma técnica eficiente, viável e processada em apenas 15 minutos. 


\section{REFERÊNCIAS}

AHMARUZZAMAN, M. Industrial wastes as low-cost potential adsorbents for the treatment of wastewater laden with heavy metals. Adv. Colloid Interface Sci., v. 166, n. 1-2, p. 3659, 2011.

AL-SHANNAG, M.; AL-QODAH, Z.; MELHEM, K. B.; QTAISHAT, M. R.; ALKASRAWI, M. Heavy Metal Ions Removal From Metal Plating Wastewater Using Electrocoagulation: Kinetic Study and Process Performance. Chem. Eng. J., v. 260, p.749-756, 2015.

BAZRAFSHAN, E.; MOHAMMADI, L.; MOGHADDAM, A. A.; MAHVI, A. H. Heavy Metals Removal From Aqueous Environments By Electrocoagulation Process - A Systematic Review. J.E. H. Scien. Eng., v. 13, n. 74, 2015.

CASQUEIRA, R. G.; TOREM, M. L.; KOHLER, H. M., The removal of zinc from liquid streams by electroflotation. Miner. Eng., n. 19, p. 1388-1392, 2006.

CONSELHO NACIONAL DO MEIO AMBIENTE. Dispõe sobre as condições e padrões de lançamento de efluentes, complementa e altera a Resolução no 357, de 17 de março de 2005, do Conselho Nacional do Meio Ambiente - CONAMA. Resolução n. 430, de 13 de maio de 2011. Diário Oficial da União. Brasília, 2011.

EL-NAAS, M. H.; HAMDAN, S. S. Removal of Hexavalent Chromium from Groundwater by Continuous Electrocoagulation. J. I. Eng. Chem., v. 20, n. 15, p. 2775-2781, 2014.

FORNARI, M. M. T. Aplicação da técnica de eletro-floculação no tratamento de efluentes de curtume. Toledo: Dissertação de Mestrado. Programa de Pós-Graduação em Engenharia Química da Universidade Estadual do Oeste do Paraná Toledo, 2007. 94 p.

KHALAF, A. M.; MUBARAK, A. A.; NOSIER, S. A. Removal of Cr (VI) by Electrocoagulation Using Vertical and Horizontal Rough Cylinder Anodes. I. J. Elec. Scien., v. 11, n. 2, p. 1601-1610, 2016.

MELLA, B. Remoção de cromo de banhos residuais de curtimento através de precipitação química e eletrocoagulação. Porto Alegre: Dissertação de Mestrado. Programa de PósGraduação em Engenharia Química da Universidade do Rio Grande do Sul, 2013.106 p.

MONTGOMERY, D. C. Design and Analysis of Experiments. New York: John Wiley and Sons, 2013. 